\title{
Semantic Product Search
}

\author{
Priyanka Nigam* \\ nigamp@amazon.com \\ Amazon \\ Palo Alto, California, USA \\ Vihan Lakshman \\ vihan@amazon.com \\ Amazon \\ Palo Alto, California, USA \\ Choon Hui Teo \\ choonhui@amazon.com \\ Amazon \\ Palo Alto, California, USA
}

\author{
Yiwei Song* \\ ywsong@amazon.com \\ Amazon \\ Palo Alto, California, USA \\ Weitian (Allen) Ding ${ }^{\dagger}$ \\ weitiand@andrew.cmu.edu \\ Carnegie Mellon University \\ Pittsburgh, Pennsylvania, USA
$\mathrm{Hao} \mathrm{Gu}$
hggu@amazon.com
Amazon
Palo Alto, California, USA

\author{
Vijai Mohan \\ vijaim@amazon.com \\ Amazon \\ Palo Alto, California, USA \\ Ankit Shingavi \\ ashingav@amazon.com \\ Amazon \\ Palo Alto, California, USA \\ Bing Yin \\ alexbyin@amazon.com \\ Amazon \\ Palo Alto, California, USA
}

\begin{abstract}
We study the problem of semantic matching in product search, that is, given a customer query, retrieve all semantically related products from the catalog. Pure lexical matching via an inverted index falls short in this respect due to several factors: a) lack of understanding of hypernyms, synonyms, and antonyms, b) fragility to morphological variants (e.g. "woman" vs. "women"), and c) sensitivity to spelling errors. To address these issues, we train a deep learning model for semantic matching using customer behavior data. Much of the recent work on large-scale semantic search using deep learning focuses on ranking for web search. In contrast, semantic matching for product search presents several novel challenges, which we elucidate in this paper. We address these challenges by a) developing a new loss function that has an inbuilt threshold to differentiate between random negative examples, impressed but not purchased examples, and positive examples (purchased items), b) using average pooling in conjunction with $n$-grams to capture short-range linguistic patterns, c) using hashing to handle out of vocabulary tokens, and d) using a model parallel training architecture to scale across 8 GPUs. We present compelling offline results that demonstrate at least 4.7\% improvement in Recall@100 and 14.5\% improvement in mean average precision (MAP) over baseline stateof-the-art semantic search methods using the same tokenization method. Moreover, we present results and discuss learnings from online A/B tests which demonstrate the efficacy of our method.
\end{abstract}

\section{CCS CONCEPTS}

- Information systems $\rightarrow$ Retrieval models and ranking; • Applied computing $\rightarrow$ Electronic commerce.

"Both authors contributed equally to this research.

${ }^{\dagger}$ This work was done while the author was at Amazon

Permission to make digital or hard copies of part or all of this work for personal or classroom use is granted without fee provided that copies are not made or distributed for profit or commercial advantage and that copies bear this notice and the full citation on the first page. Copyrights for third-party components of this work must be honored.

For all other uses, contact the owner/author(s).

KDD '19, August 4-8, 2019, Anchorage, AK, USA

(C) 2019 Copyright held by the owner/author(s)

ACM ISBN 978-1-4503-6201-6/19/08.

https://doi.org/10.1145/3292500.3330759

\section{KEYWORDS}

Semantic Matching, Product Search, Neural Information Retrieval

\section{ACM Reference Format:}

Priyanka Nigam, Yiwei Song, Vijai Mohan, Vihan Lakshman, Weitian (Allen) Ding, Ankit Shingavi, Choon Hui Teo, Hao Gu, and Bing Yin. 2019. Semantic Product Search. In The 25th ACM SIGKDD Conference on Knowledge Discovery and Data Mining (KDD '19), August 4-8, 2019, Anchorage, AK, USA. ACM, New York, NY, USA, 10 pages. https://doi.org/10.1145/3292500.3330759

\section{INTRODUCTION}

At a high level, as shown in Figure 1, a product search engine works as follows: a customer issues a query, which is passed to a lexical matching engine (typically an inverted index [17, 30]) to retrieve all products that contain words in the query, producing a match set. The match set passes through stages of ranking, wherein top results from the previous stage are re-ranked before the most relevant items are finally displayed. It is imperative that the match set contain a relevant and diverse set of products that match the customer intent in order for the subsequent rankers to succeed. However, inverted index-based lexical matching falls short in several key aspects:

- Lack of understanding of hypernyms (generalizations of words), synonyms (different words with the same meaning), and antonyms (words that have opposite meanings). For example, "sneakers" might match the intent of the query running shoes, but may not be retrieved. Similarly, a "red dress" matches the semantic intent of the query burgundy dress and yet is not retrieved by a lexical matching engine. Finally, "latex free examination gloves" do not match the intent of the query latex examination gloves, and yet are retrieved simply because all the words in the query are also present in the product title and description.

- Fragility to morphological variants (e.g. "woman" vs. "women"). One can address this issue to some extent by applications of stemming or lemmatization. However, stemming algorithms are often imperfect and lead to information loss and errors. For instance, a stemmer that truncates nouns into their singular form might transform the query "reading glasses" into "reading glass" and fail to return relevant 
results. To be viable in production, these approaches typically require numerous hand-crafted rules that may become obsolete and fail to generalize to multiple languages.

- Sensitivity to spelling errors. According to some estimates of web search logs $[3,4], 10-15 \%$ of queries are misspelled. This leads to customer confusion (why are there no results for the query "rred drress"?) and frustration. While modern spell-correction methods can reduce the problem, a matching engine that handles spelling errors would be simpler.

In this paper, we address the question: Given rich customer behavior data, can we train a deep learning model to retrieve matching products in response to a query? Intuitively, there is reason to believe that customer behavior logs contain semantic information; customers who are intent on purchasing a product circumvent the limitations of lexical matching by query reformulation or by deeper exploration of the search results. The challenge is the sheer magnitude of the data as well as the presence of noise, a challenge that modern deep learning techniques address very effectively.

Product search is different from web search as the queries tend to be shorter and the positive signals (purchases) are sparser than clicks. Models based on conversion rates or click-through-rates may incorrectly favor accessories (like a phone cover) over the main product (like a cell phone). This is further complicated by shoppers maintaining multiple intents during a single search session: a customer may be looking for a specific television model while also looking for accessories for this item at the lowest price and browsing additional products to qualify for free shipping. A product search engine should reduce the effort needed from a customer with a specific mission (narrow queries) while allowing shoppers to explore when they are looking for inspiration (broad queries).

As mentioned, product search typically operates in two stages: matching and ranking. Products that contain words in the query $\left(Q_{i}\right)$ are the primary candidates. Products that have prior behavioral associations (products bought or clicked after issuing a query $Q_{i}$ ) are also included in the candidate set. The ranking step takes these candidates and orders them using a machine-learned rank function to optimize for customer satisfaction and business metrics.

We present a neural network trained with large amounts of purchase and click signals to complement a lexical search engine in ad hoc product retrieval. Our first contribution is a loss function with a built-in threshold to differentiate between random negative, impressed but not purchased, and purchased items. Our second contribution is the empirical result that recommends average pooling in combination with $n$-grams that capture short-range linguistic patterns instead of more complex architectures. Third, we show the effectiveness of consistent token hashing in Siamese networks for zero-shot learning and handling out of vocabulary tokens.

In Section 2, we highlight related work. In Section 3, we describe our model architecture, loss functions, and tokenization techniques including our approach for unseen words. We then introduce the readers to the data and our input representations for queries and products in Section 4. Section 5 presents the evaluation metrics and our results. We provide implementation details and optimizations to efficiently train the model with large amounts of data in Section 6. Finally, we conclude in Section 7 with a discussion of future work.

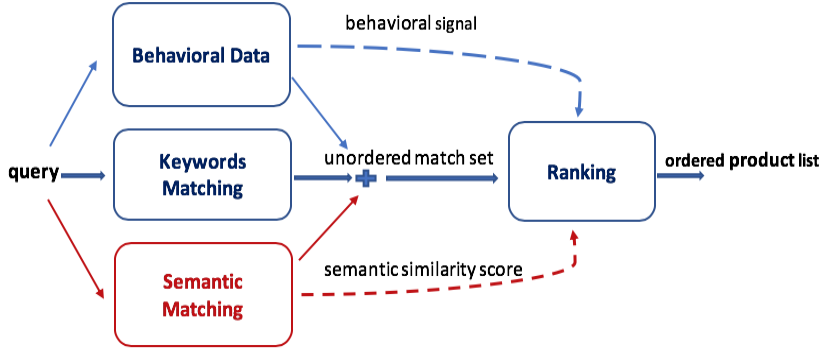

Figure 1: System architecture for augmenting product matching using semantic matching

\section{RELATED WORK}

There is a rich literature in natural language processing (NLP) and information retrieval (IR) on capturing the semantics of queries and documents. Word2vec [18] garnered significant attention by demonstrating the use of word embeddings to capture semantic structure; synonyms cluster together in the embedding space. This technique was successfully applied to document ranking for web search with the DESM model [20]. Building from the ideas in word2vec, Diaz et al. [6] trained neural word embeddings to find neighboring words to expand queries with synonyms. Ultimately, based on these recent advancements and other key insights, the state-of-the-art models for semantic search can generally be classified into three categories:

(1) Latent Factor Models: Nonlinear matrix completion approaches that learn query and document-level embeddings without using their content.

(2) Factorized Models: Separately convert queries and documents to low-dimensional embeddings based on content.

(3) Interaction Models: Build interaction matrices between the query and document text and use neural networks to mine patterns from the interaction matrix

Deerwester et al. [5] introduced Latent Semantic Analysis (LSA), which computes a low-rank factorization of a term-document matrix to identify semantic concepts and was further refined by $[1,7]$ and extended by ideas from Latent Dirichlet Allocation (LDA) [2] in [27]. In 2013, Huang et al. [11] published the seminal paper in the space of factorized models by introducing the Deep Semantic Similarity Model (DSSM). Inspired by LSA and Semantic Hashing [23], DSSM involves training an end-to-end deep neural network with a discriminative loss to learn a fixed-width representation for queries and documents. Fully connected units in the DSSM architecture were subsequently replaced with Convolutional Neural Networks (CNNs) [10, 24] and Recurrent Neural Networks (RNNs) [21] to respect word ordering. In an alternate approach, which articulated the idea of interaction models, Guo et al. [9] introduced the Deep Relevance Matching Model (DRMM) which leverages an interaction matrix to capture local term matching within neural approaches which has been successfully extended by MatchPyramid [22] and other techniques [12-14, 26, 29]. Nevertheless, these interaction methods require memory and computation proportional to the number of words in the document and hence are prohibitively expensive for online inference. In addition, Duet [19] combines 
the approaches of DSSM and DRMM to balance the importance of semantic and lexical matching. Despite obtaining state-of-the-art results for ranking, these methods report limited success on ad hoc retrieval tasks [19] and only achieve a sub-50\% Recall@100 and MAP on our product matching dataset, as shown with the ARC-II and Match Pyramid baselines in Table 5.

While we frequently evaluate our hypotheses on interaction matrix-based methods, we find that a factorized model architecture achieves comparable performance while only requiring constant memory per product. Hence, we only present our experiments as it pertains to factorized models in this paper. Although latent factor models improve ranking metrics due to their ability to memorize associations between the query and the product, we exclude it from this paper as we focus on the matching task. Our choice of model architecture was informed by empirical experiments while constrained by the cost per query and our ability to respond within 20 milliseconds for thousands of queries per second.

\section{MODEL}

\subsection{Neural Network Architecture}

Our neural network architecture is shown in Figure 2. As in the distributed arm of the Duet model, our first model component is an embedding layer that consists of $|V| \times N$ parameters where $V$ is the vocabulary and $N$ is the embedding dimension. Each row corresponds to the parameters for a word. Unlike Duet, we share our embeddings across the query and product. Intuitively, sharing the embedding layer in a Siamese network works well, capturing local word-level matching even before training these networks. Our experiments in Table 7 confirm this intuition. We discuss the specifics of our query and product representation in Section 4.

To generate a fixed length embedding for the query $\left(E^{Q}\right)$ and the product $\left(E^{P}\right)$ from individual word embeddings, we use average pooling after observing little difference $(<0.5 \%)$ in both MAP and Recall@100 relative to recurrent approaches like LSTM and GRU (see Table 2). Average pooling also requires far less computation, reducing training time and inference latency. We reconciled this departure from state-of-the-art solutions for Question Answering and other NLP tasks through an analysis that showed that, unlike web search, both query and product information tend to be shorter, without long-range dependencies. Additionally, product search queries do not contain stop words and typically require every query word (or its synonym) to be present in the product.

Queries typically have fewer words than the product content. Because of this, we observed a noticeable difference in the magnitude of query and product embeddings. This was expected as the query and the product models were shared with no additional parameters to account for this variance. Hence, we introduced Batch Normalization layers [15] after the pooling layers for the query and the product arms. Finally, we compute the cosine similarity between $E^{Q}$ and $E^{P}$. During online A/B testing, we precompute $E^{P}$ for all the products in the catalog and use a $k$-Nearest Neighbors algorithm to retrieve the most similar products to a given query $Q_{i}$.

\subsection{Loss Function}

A critical decision when employing a vector space model is defining a match, especially in product search where there is an important

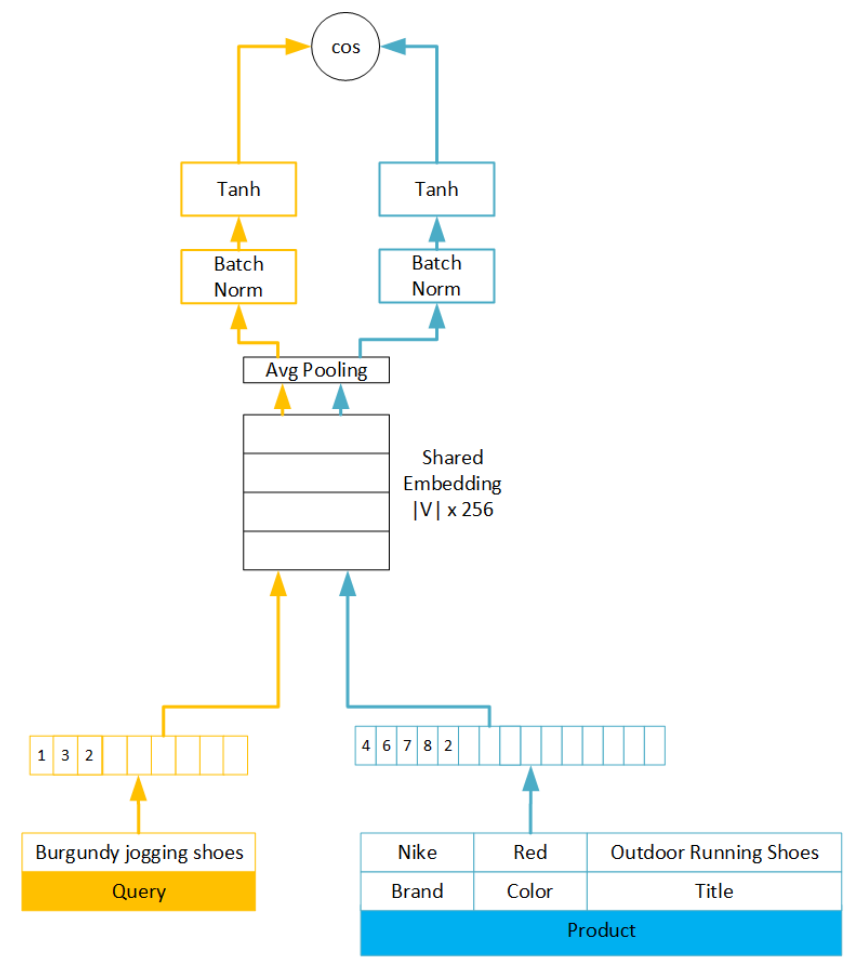

Figure 2: Illustration of neural network architecture used for semantic search

tradeoff between precision and recall. For example, accessories like mounts may also be relevant for the query "led tv."

Pruning results based on a threshold is a common practice to identify the match set. Pointwise loss functions, such as mean squared error (MSE) or mean absolute error (MAE), require an additional step post-training to identify the threshold. Pairwise loss functions do not provide guarantees on the magnitude of scores (only on relative ordering) and thus do not work well in practice with threshold-based pruning. Hence, we started with a pointwise 2-part hinge loss function as shown in Equation (1) that maximizes the similarity between the query and a purchased product while minimizing the similarity between a query and random products. Define $\hat{y}:=\cos \left(E^{Q}, E^{P}\right)$, and let $y=1$ if product $P$ is purchased in response to query $Q$, and $y=0$ otherwise. Furthermore let $\ell_{+}(y):=\left(-\min \left(0, y-\epsilon_{+}\right)\right)^{m}$, and $\ell_{-}(y):=\max \left(0, y-\epsilon_{-}\right)^{m}$ for some predefined thresholds $\epsilon_{+}$and $\epsilon_{-}$and $m \in\{1,2\}$. The two part hinge loss can be defined as

$$
L(\hat{y}, y):=y \cdot \ell_{+}(\hat{y})+(1-y) \cdot \ell_{-}(\hat{y})
$$

Intuitively, the loss ensures that when $y=0$ then $\hat{y}$ is less than $\epsilon_{-}$ and when $y=1$ then $\hat{y}$ is above $\epsilon_{+}$. After some empirical tuning on a validation set, we set $\epsilon_{+}=0.9$ and $\epsilon_{-}=0.2$.

As shown in Table 1, the 2-part hinge loss improved offline matching performance by more than $2 \mathrm{X}$ over the MSE baseline. However in Figure 3, a large overlap in score distribution between positives and negatives can be seen. Furthermore, the score distribution for negatives appeared bimodal. After manually inspecting the 


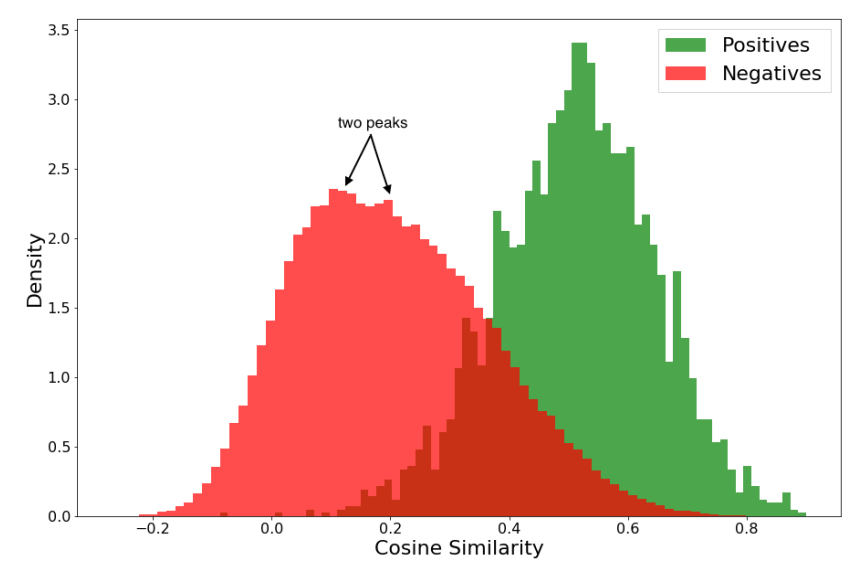

Figure 3: Score distribution histogram shows large overlap for positives (right) and negatives (left) along with a bimodal distribution for negatives when using the 2-part hinge

negative training examples that fell in this region, we uncovered that these were products that were impressed but not purchased by the customer. From a matching standpoint, these products are usually valid results to show to customers. To improve the model's ability to distinguish positives and negatives considering these two classes of negatives, we introduced a 3-part hinge loss:

$$
L(\hat{y}, y):=I^{+}(y) \cdot \ell_{+}(\hat{y})+I^{-}(y) \cdot \ell_{-}(\hat{y})+I^{0}(y) \cdot \ell_{0}(\hat{y})
$$

where $I^{+}(y), I^{-}(y)$, and $I^{0}(y)$ denote indicators signifying if the product $P$ was purchased, not impressed and not purchased, and impressed (but not purchased) in response to the query $Q$, respectively, and $\ell_{0}(\hat{y}):=\max \left(0, \hat{y}-\epsilon_{0}\right)^{m}$. Based on the 2-part hinge score distribution, $\epsilon_{0}$ was set to 0.55 with $\epsilon_{+}=0.9$ and $\epsilon_{-}=0.2$ as before. The effectiveness of this strategy can be seen in Figure 4, where one can observe a clear separation in scores between random and impressed negatives vs positives.

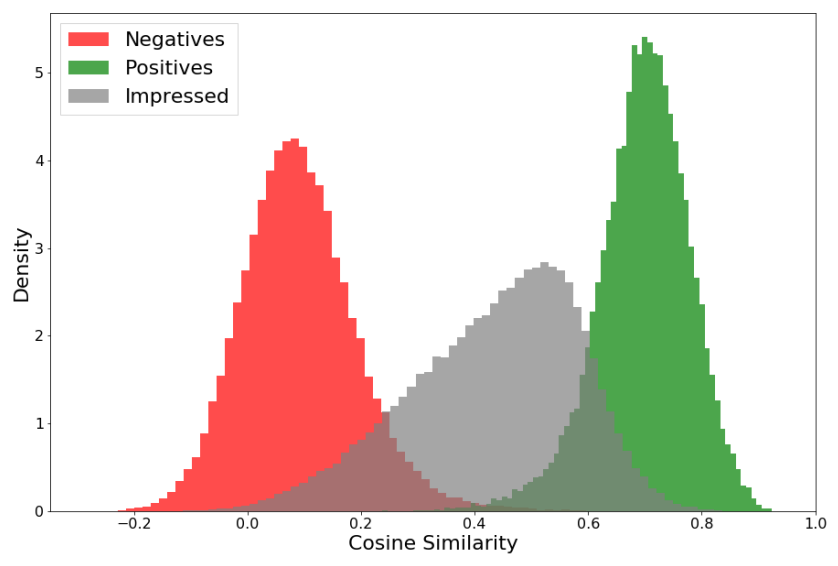

Figure 4: Score distribution shows clear separation between purchased (right), seen but not purchased (center), and irrelevant products (left) when using the 3-part hinge

\subsection{Tokenization Methods}

In this section, we describe our tokenization methodology, or the procedure by which we break a string into a sequence of smaller components such as words, phrases, sub-words, or characters. We combine word unigram, word n-gram, and character trigram features into a bag of $\mathrm{n}$-grams and use hashing to handle the large vocabulary size, similar to the fastText approach [16].

3.3.1 Word Unigram. This is the basic form of tokenization where the input query or product title is tokenized into a list of words. For example, the word unigrams of "artistic iphone 6s case" are ["artistic", "iphone", "6s", "case"].

3.3.2 Word N-gram. In a bag of words model like ours, word unigrams lose word ordering. Instead of using LSTMs or CNNs to address this issue, we opted for $n$-grams as in [25]. For example, the word bigrams of "artistic iphone 6s case" are ["artistic\#iphone", "iphone\#6s", "6s\#case"] and the trigrams are ["artistic\#iphone\#6s", "iphone\#6s\#case"]. These $n$-grams capture phrase-level information; for example if "for iphone" exists in the query, the model can infer that the customer's intention is to search for iphone accessories rather than iphone - an intent not captured by a unigram model.

3.3.3 Character Trigram. Character trigram embeddings were proposed by the DSSM paper [11]. The string is broken into a list of all three-character sequences. For the example "artistic iphone $6 \mathrm{~s}$ case", the character trigrams are ["\#ar", "art", "rti", "tis", "ist", "sti", "tic", "ic\#", "c\#i", "\#ip", "iph", "pho", "hon", "one", "ne\#", "e\#6", "\#6s", "6s\#", "s\#c", "\#ca", "cas", "ase", "se\#"]. Character trigrams are robust to typos ("iphione" and "iphonr") and handle compound words ("amazontv" and "firetvstick") naturally. Another advantage in our setting is the ability to capture similarity of model parts and sizes.

3.3.4 Handling Unseen Words. It is computationally infeasible to maintain a vocabulary that includes all the possible word $n$-grams as the dictionary size grows exponentially with $n$. Thus, we maintain a "short" list of several tens or hundreds of thousands of $n$-grams based on token frequency. A common practice for most NLP applications is to mask the input or use the embedding from the $0^{\text {th }}$ location when an out-of-vocabulary word is encountered. Unfortunately, in Siamese networks, assigning all unknown words to the same shared embedding location results in incorrectly mapping two different out-of-vocabulary words to the same representation. Hence, we experimented with using the "hashing trick" [28] popularized by Vowpal Wabbit to represent higher order $n$-grams that are not present in the vocabulary. In particular, we hash out-ofvocabulary tokens to additional embedding bins. The combination of using a fixed hash function and shared embeddings ensures that unseen tokens that occur in both the query and document map to the same embedding vector. During our initial experiments with a bin size of 10,000, we noticed that hashing collisions incorrectly promoted irrelevant products for queries, led to overfitting, and did not improve offline metrics. However, setting a bin size 5-10 times larger than the vocabulary size improved the recall of the model.

3.3.5 Combining Tokenizations. There are several ways to combine the tokens from these tokenization methods. One could create separate embeddings for unigrams, bigrams, character trigrams, etc. and compute a weighted sum over the cosine similarity of 
these $n$-gram projections. But we found that the simple approach of combining all tokens in a single bag-of-tokens performs well. We conclude this section by referring the reader to Figure 5, which walks through our tokenization methods for the example "artistic iphone 6s case". In Table 6, we show example queries and products retrieved to highlight the efficacy of our best model to understand synonyms, intents, spelling errors and overall robustness.

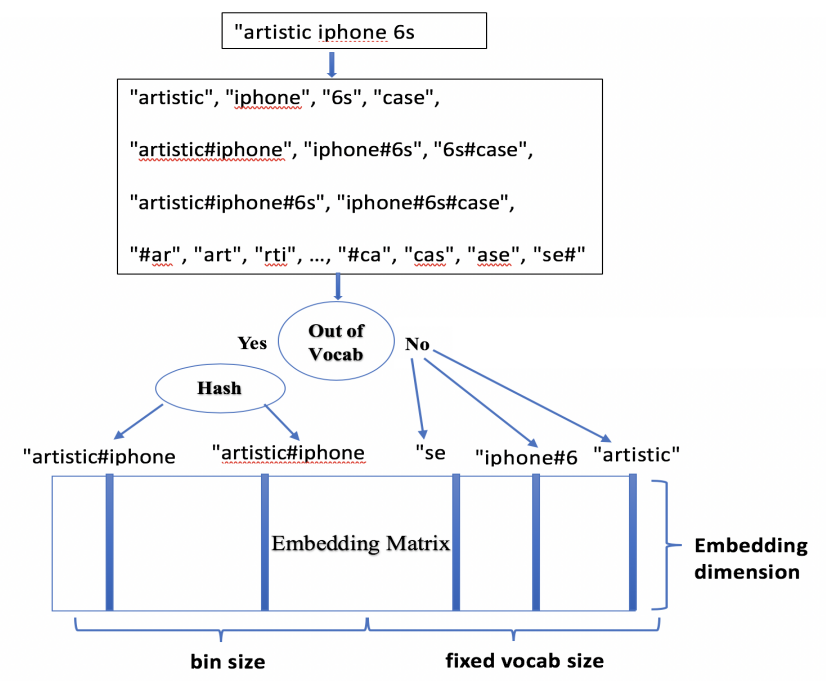

Figure 5: Aggregation of different tokenization methods illustrated with the processing of "artistic iphone $6 \mathrm{~s}$ case"

\section{DATA}

We use 11 months of search logs as training data and 1 month as evaluation. We sample 54 billion query-product training pairs. We preprocess these sampled pairs to 650 million rows by grouping the training data by query-product pairs over the entire time period and using the aggregated counts as weights for the pairs. We also decrease the training time by $3 \mathrm{X}$ by preprocessing the training data into tokens and using mmap to store the tokens. More details on our best practices for reducing training time can be found in Section 6 .

For a given customer query, each product is in exactly one of three categories: purchased, impressed but not purchased, or random. For each query, we target a ratio of 6 impressed and 7 random products for every query-product purchase. We sample this way to train the model for both matching and ranking, although in this paper we focus on matching. Intuitively, matching should differentiate purchased and impressed products from random ones; ranking should differentiate purchased products from impressed ones.

We choose the most frequent words to build our vocabulary, referred to as $|V|$. Each token in the vocabulary is assigned a unique numeric token id, while remaining tokens are assigned 0 or a hashing based identifier. Queries are lowercased, split on whitespace, and converted into a sequence of token ids. We truncate the query tokens at the 99th percentile by length. Token vectors that are smaller than the predetermined length are padded to the right.

Products have multiple attributes, like title, brand, and color, that are material to the matching process. We evaluated architectures to embed every attribute independently and concatenate them to obtain the final product representation. However, large variability in the accuracy and availability of structured data across products led to $5 \%$ lower recall than simply concatenating the attributes. Hence, we decided to use an ordered bag of words of these attributes.

\section{EXPERIMENTS}

In this section we describe our metrics, training procedure, and the results, including the impact of our method in production.

\subsection{Metrics}

We define two evaluation subtasks: matching and ranking.

(1) Matching: The goal of the matching task is to retrieve all relevant documents from a large corpus for a given query. In order to measure the matching performance, we first sample a set of $20 \mathrm{~K}$ queries. We then evaluate the model's ability to recall purchased products from a sub-corpus of 1 million products for those queries. Note that the 1 million product corpus contains purchased and impressed products for every query from the evaluation period as well as additional random negatives. We tune the model hyperparameters to maximize Recall@100 and Mean Average Precision (MAP).

(2) Ranking: The goal of this task is to order a set of documents by relevance, defined as purchase count conditioned on the query. The set of documents contains purchased and impressed products. We report standard information retrieval ranking metrics, such as Normalized Discounted Cumulative Gain (NDCG) and Mean Reciprocal Rank (MRR).

\subsection{Results}

In this section, we present the durable learnings from thousands of experiments. We fix the embedding dimension to 256, weight matrix initialization to Xavier initialization [8], batch size to 8192 , and the optimizer to ADAM with the configuration $\alpha=0.001, \beta_{1}=$ $0.9, \beta_{2}=0.999, \epsilon=10^{-8}$ for all the results presented. We refer to the hinge losses defined in Section 3.2 with $m=1$ and $m=2$ as the L1 and L2 variants respectively. Unigram tokenization is used in Table 1 and Table 2, as the relative ordering of results does not change with other more sophisticated tokenizations.

We present the results of different loss functions in Table 1. We see that the L2 variant of each loss consistently outperforms the L1. We hypothesize that L2 variants are robust to outliers in cosine similarity. The 3-part hinge loss outperforms the 2-part hinge loss in matching metrics in all experiments although the two loss functions have similar ranking performance. By considering impressed negatives, whose text is usually more similar to positives than negatives, separately from random negatives in the 3-part hinge loss, the scores for positives and random negatives become better separated, as shown in Section 3.2. The model can better differentiate between positives and random negatives, improving Recall and MAP. Because the ranking task is not distinguishing between relevant and random products but instead focuses on ordering purchased and impressed products, it is not surprising that the 2-part and 3-part loss functions have similar performance.

In Table 2, we present the results of using LSTM, GRU, and averaging to aggregate the token embeddings. Averaging performs 
Table 1: Loss Function Experiments using Unigram Tokenization and Average Pooling

\begin{tabular}{l|cccc|cc}
\hline Loss & Recall & MAP & Matching NDCG & Matching MRR & Ranking NDCG & Ranking MRR \\
\hline BCE & $\mathbf{0 . 5 8 6}$ & $\mathbf{0 . 4 8 6}$ & $\mathbf{0 . 6 9 5}$ & $\mathbf{0 . 4 7 3}$ & $\mathbf{0 . 7 1 1}$ & $\mathbf{0 . 9 5 4}$ \\
MAE & 0.044 & 0.020 & 0.275 & 0.192 & 0.611 & 0.905 \\
MSE & 0.238 & 0.144 & 0.490 & 0.377 & 0.680 & 0.948 \\
\hline 2 Part L1 & 0.485 & 0.384 & 0.694 & 0.472 & 0.742 & 0.966 \\
3 Part L1 & $\mathbf{0 . 6 9 1}$ & $\mathbf{0 . 6 1 6}$ & $\mathbf{0 . 7 6 2}$ & $\mathbf{0 . 5 3 6}$ & $\mathbf{0 . 7 6 0}$ & $\mathbf{0 . 9 7 1}$ \\
\hline 2 Part L2 & 0.651 & 0.576 & 0.768 & 0.549 & $\mathbf{0 . 7 7 6}^{*}$ & $\mathbf{0 . 9 7 3}^{*}$ \\
3 Part L2 & $\mathbf{0 . 7 3 5}^{\text {* }}$ & $\mathbf{0 . 6 6 4}^{\text {* }}$ & $\mathbf{0 . 7 9 1}^{\text {* }}$ & $\mathbf{0 . 5 9 1}^{\text {* }}$ & 0.772 & $\mathbf{0 . 9 7 3}^{*}$ \\
\hline
\end{tabular}

Table 2: Token Embedding Aggregation Experiments using Unigram Tokenization

\begin{tabular}{ll|cccc|cc}
\hline Loss & Pooling & Recall & MAP & Matching NDCG & Matching MRR & Ranking NDCG & Ranking MRR \\
\hline \multirow{3}{*}{ MSE } & ave & $\mathbf{0 . 2 3 8}$ & $\mathbf{0 . 1 4 4}$ & $\mathbf{0 . 4 9 0}$ & $\mathbf{0 . 3 7 7}$ & 0.680 & 0.948 \\
& gru & 0.105 & 0.052 & 0.431 & 0.348 & 0.700 & 0.951 \\
& lstm & 0.102 & 0.048 & 0.404 & 0.286 & $\mathbf{0 . 6 9 7}$ & $\mathbf{0 . 9 4 8}$ \\
\hline \multirow{3}{*}{3 Part L1 } & ave & $\mathbf{0 . 6 9 1}$ & $\mathbf{0 . 6 1 6}$ & $\mathbf{0 . 7 6 2}$ & $\mathbf{0 . 5 3 6}$ & $\mathbf{0 . 7 6 0}$ & $\mathbf{0 . 9 7 1}$ \\
& gru & 0.651 & 0.574 & 0.701 & 0.376 & 0.727 & 0.965 \\
& lstm & 0.661 & 0.588 & 0.730 & 0.469 & 0.739 & 0.964 \\
\hline \multirow{3}{*}{3 Part L2 } & ave & 0.735 & 0.664 & $\mathbf{0 . 7 9 1}^{*}$ & $\mathbf{0 . 5 9 1}^{*}$ & 0.772 & 0.973 \\
& gru & $\mathbf{0 . 7 3 9}^{*}$ & 0.659 & 0.777 & 0.578 & $\mathbf{0 . 7 7 5}^{*}$ & $\mathbf{0 . 9 7 5}^{*}$ \\
& lstm & 0.738 & $\mathbf{0 . 6 6 6 *}$ & 0.767 & 0.527 & $\mathbf{0 . 7 7 5}^{*}$ & $\mathbf{0 . 9 7 6}^{*}$ \\
\hline
\end{tabular}

Table 3: Tokenization Experiments with Average Pooling and 3 Part L2 Hinge Loss

\begin{tabular}{l|cccc|cc}
\hline Tokenization & Recall & MAP & Matching NDCG & Matching MRR & Ranking NDCG & Ranking MRR \\
\hline Char Trigrams & 0.673 & 0.586 & 0.718 & 0.502 & 0.741 \\
Unigrams & 0.735 & 0.664 & 0.791 & 0.591 & 0.772 & 0.955 \\
Unigrams+Bigrams & 0.758 & 0.696 & 0.784 & 0.577 & 0.768 & 0.974 \\
Unigrams+Bigrams+Char Trigrams & $\mathbf{0 . 7 6 4}$ & $\mathbf{0 . 7 0 7}$ & $\mathbf{0 . 8 0 0}$ & $\mathbf{0 . 6 1 5}$ & $\mathbf{0 . 7 9 4}$ & $\mathbf{0 . 9 7 8}$ \\
\hline Unigrams+OOV & 0.752 & 0.694 & 0.799 & 0.633 & 0.791 & 0.978 \\
Unigrams+Bigrams+OOV & 0.789 & 0.741 & 0.790 & 0.610 & 0.776 & 0.979 \\
Unigrams+Bigrams+Char Trigrams+OOV & $\mathbf{0 . 7 9 4}^{*}$ & $\mathbf{0 . 7 4 5}$ & $\mathbf{0 . 8 1 0}$ & $\mathbf{0 . 6 5 9}^{*}$ & $\mathbf{0 . 7 9 4}^{*}$ & $\mathbf{0 . 9 8 0}$ \\
\hline Unigrams(500K) & 0.745 & 0.683 & 0.799 & $\mathbf{0 . 6 2 9}$ & 0.784 & 0.975 \\
Word Unigrams(125K)+OOV(375K) & $\mathbf{0 . 7 5 3}$ & $\mathbf{0 . 6 9 4}$ & $\mathbf{0 . 8 0 4}$ & 0.612 & $\mathbf{0 . 7 8 8}$ & $\mathbf{0 . 9 7 9}$ \\
\hline
\end{tabular}

similar to or slightly better than recurrent units with significantly less training time. As mentioned in Section 3.1, in the product search setting, queries and product titles tend to be relatively short, so averaging is sufficient to capture the short-range dependencies that exist in queries and product titles. Furthermore, recurrent methods are more expressive but introduce specialization between the query and title. Consequently, local word-level matching between the query and the product title may not be not captured as well.

In Table 3, we compare the performance of using different tokenization methods. We use average pooling and the 3-part L2 hinge loss. For each tokenization method, we select the top $k$ terms by frequency in the training data. Unless otherwise noted, $k$ was set to $125 \mathrm{~K}, 25 \mathrm{~K}, 64 \mathrm{~K}$, and $500 \mathrm{~K}$ for unigrams, bigrams, character trigrams, and out-of-vocabulary (OOV) bins respectively. It is worth noting that using only character trigrams, which was an essential component of DSSM[11], has competitive ranking but not matching performance compared to unigrams. Adding bigrams improves matching performance as bigrams capture short phrase-level information that is not captured by averaging unigrams. For example, the unigrams for "chocolate milk" and "milk chocolate" are the same although these are different products. Additionally including character trigrams improves the performance further as character trigrams provide generalization and robustness to spelling errors.

Adding OOV hashing improves the matching performance as it allows better generalization to infrequent or unseen terms, with the caveat that it introduces additional parameters. To differentiate between the impact of additional parameters and OOV hashing, the last two rows in Table 3 compare $500 \mathrm{~K}$ unigrams to $125 \mathrm{~K}$ unigrams and $375 \mathrm{~K}$ OOV bins. These models have the same number of parameters, but the model with OOV hashing performs better. 
In Table 4, we present the results of using batch normalization, layer normalization, or neither on the aggregated query and product embeddings. The "Query Sorted" column refers to whether all positive, impressed, and random negative examples for a single query appear together or are shuffled throughout the data. The best matching performance is achieved using batch normalization and shuffled data. Using sorted data has a significantly negative impact on performance when using batch normalization but not when using layer normalization. Possibly, the batch estimates of mean and variance are highly biased when using sorted data.

Finally, in Table 5, we compare the results of our model to four baselines: DSSM [11], Match Pyramid [22], ARC-II [10], and our model with frozen, randomly initialized embeddings. We only use word unigrams or character trigrams in our model, as it is not immediately clear how to extend the bag-of-tokens approach to methods that incorporate ordering. We compare the performance of using the 3-part L2 hinge loss to the original loss presented for each model. Across all baselines, matching performance of the model improves using the 3-part L2 hinge loss. ARC-II and Match Pyramid ranking performance is similar or lower when using the 3-part loss. Ranking performance improves for DSSM, possibly because the original approach uses only random negatives to approximate the softmax normalization. More complex models, like Match Pyramid and ARC-II, had significantly lower matching and ranking performance while taking significantly longer to train and evaluate. These models are also much harder to tune and tend to overfit.

The embeddings in our model are trained end-to-end. Previous experiments using other methods, including Glove and word2vec, to initialize the embeddings yielded poorer results than end-to-end training. When comparing our model with randomly initialized to one with trained embeddings, we see that end-to-end training results in over a 3X improvement in Recall@100 and MAP.

\subsection{Online Experiments}

We ran a total of three online match set augmentation experiments on a large e-commerce website across three categories: toys and games, kitchen, and pets. In all experiments, the conversion rate, revenue, and other key performance indicators (KPIs) statistically significantly increased. One challenge we faced with our semantic search solution was weeding out irrelevant results to meet the precision bar for production search quality. To boost the precision of the final results, we added guard rails through additional heuristics and ranking models to filter irrelevant products. A qualitative analysis of the augmented search results coupled with an increase in relevant business metrics provided us with compelling evidence that this approach contributed to our goal of helping customers effortlessly complete their shopping missions.

\section{TRAINING ACCELERATION}

During our offline experiments, we saw an average of $10 \%$ improvement in matching metrics by increasing the data from 200 million to 1.2 billion query-product pairs. In this section, we describe our multi-GPU training techniques for efficiently handling these larger datasets. Most parameters for this model lie in the embedding layer and hence data parallel training approaches have high communication overhead. Furthermore data parallel training limits the embedding matrix size as the model must fit in a single GPU. The simplicity of averaging pooling combined with the separability of the Siamese architecture allow us to use model parallel training to increase the throughput. Letting $k$ represent the embedding dimension and $n$ represent the number of GPUs, the similarity function of our model is shown in equation 3 . The embedding matrix is split among the GPUs along the embedding dimension. The input is sent to all GPUs to look up the partial token embeddings and average them. Sending the input to all GPUs tends to be inexpensive as the number of tokens is small in comparison with the token embeddings or the embedding matrix. Simply concatenating the partial average embeddings across GPUs requires $O(2 k)$ communication of floating point numbers per example in both forward and backward propagation. Equation 6 and 7 show how instead the cosine similarity can be decomposed to transmit only the partial-sums and partial-sum-of-squares. With this decomposition, we incur a constant communication cost of 3 scalars per GPU.

$$
\begin{gathered}
\operatorname{Sim}(Q, P)=\cos \left(E^{Q}, E^{P}\right) \\
\cos (a, b)=\frac{a \cdot b}{\|a\|_{2}\|b\|_{2}}=\frac{\sum_{i=1}^{k} a_{i} \cdot b_{i}}{\sqrt{\sum_{i=1}^{k} a_{i}^{2}} \sqrt{\sum_{i=1}^{k} b_{i}^{2}}}
\end{gathered}
$$

Splitting the cosine similarity computation across $n$ GPUs:

$$
\begin{aligned}
r & =k / n \\
\sum_{i=1}^{k} a_{i} \cdot b_{i} & =\sum_{j=1}^{n} \sum_{l=1}^{r} a_{r(j-1)+l} \cdot b_{r(j-1)+l} \\
\sum_{i=1}^{k} a_{i}^{2} & =\sum_{j=1}^{n} \sum_{l=1}^{r} a_{r(j-1)+l}^{2}
\end{aligned}
$$

Results from these experiments are shown in Figure 6. We ran experiments on a single AWS p3.16xlarge machine with 8 NVIDIA Tesla V100 GPUs (16GB), Intel Xeon E5-2686v4 processors, and $488 \mathrm{~GB}$ of RAM. The training was run 5 times with 10 million examples. The median time, scaled to 1 billion examples, is reported.

To achieve scaling, we had to ensure that the gradient variables were placed on the same GPUs as their corresponding operations. This allows greater distribution of memory usage and computation across all GPUs. Unsurprisingly, splitting the model across GPUs for smaller embedding dimensions (<256) increases the overall training time. But beyond an embedding dimension of 512, the communication overhead is less than the additional computational power. Note that the training time is almost linear at a constant embedding dimension per GPU. In other words, training with an embedding dimension of 2048 on 2 GPUs and an embedding dimension of 1024 on 1 GPU have similar speeds. In Figure 6 , this is shown by the dotted lines connecting points with the same embedding dimension per GPU. With ideal scaling, the lines would be horizontal. 
Table 4: Normalization Layer Experiments

\begin{tabular}{ll|cccc|cc}
\hline Query Sorted & Normalization & Recall & MAP & Matching NDCG & Matching MRR & Ranking NDCG & Ranking MRR \\
\hline \multirow{4}{*}{$\mathrm{T}$} & batch & 0.730 & 0.663 & 0.763 & 0.553 & 0.751 & 0.970 \\
& layer & $\mathbf{0 . 7 8 2}$ & $\mathbf{0 . 7 3 3}$ & $\mathbf{0 . 8 1 7}^{*}$ & $\mathbf{0 . 6 4 9}^{*}$ & $\mathbf{0 . 8 1 2}^{*}$ & $\mathbf{0 . 9 8 2}^{*}$ \\
\hline \multirow{3}{*}{$\mathrm{F}$} & none & 0.780 & 0.722 & 0.798 & 0.616 & 0.799 & 0.976 \\
& batch & $\mathbf{0 . 7 9 4}^{*}$ & $\mathbf{0 . 7 4 5 ^ { * }}$ & $\mathbf{0 . 8 1 0}$ & $\mathbf{0 . 6 5 9}$ & 0.794 & $\mathbf{0 . 9 8 0}$ \\
& layer & 0.791 & 0.743 & 0.807 & 0.629 & 0.797 & $\mathbf{0 . 9 8 0}$ \\
& none & 0.784 & 0.728 & 0.803 & 0.639 & $\mathbf{0 . 8 0 3}$ & 0.976 \\
\hline
\end{tabular}

Table 5: Comparison with Baselines

\begin{tabular}{|c|c|c|c|c|c|c|c|c|}
\hline Model & Loss & Tokenization & Recall & MAP & $\begin{array}{c}\text { Matching } \\
\text { NDCG }\end{array}$ & $\begin{array}{c}\text { Matching } \\
\text { MRR }\end{array}$ & $\begin{array}{c}\text { Ranking } \\
\text { NDCG }\end{array}$ & $\begin{array}{c}\text { Ranking } \\
\text { MRR }\end{array}$ \\
\hline \multirow{2}{*}{ Our Model } & 3 Part L2 & Char Trigrams & 0.673 & 0.586 & 0.718 & 0.502 & 0.741 & 0.955 \\
\hline & 3 Part L2 & Unigrams & 0.735 & 0.664 & 0.791 & 0.591 & 0.772 & 0.973 \\
\hline \multirow{2}{*}{$\begin{array}{l}\text { Our Model } \\
\text { (Random Emb) }\end{array}$} & 3 Part L2 & Char Trigrams & 0.268 & 0.149 & 0.291 & 0.075 & 0.426 & 0.792 \\
\hline & 3 Part L2 & Unigrams & 0.207 & 0.107 & 0.249 & 0.052 & 0.412 & 0.778 \\
\hline \multirow{4}{*}{ DSSM [11] } & Crossentropy & Char Trigrams & 0.647 & 0.537 & 0.576 & 0.278 & 0.589 & 0.903 \\
\hline & 3 Part L2 & Char Trigrams & 0.662 & 0.568 & 0.726 & 0.557 & 0.745 & 0.956 \\
\hline & Crossentropy & Unigrams & 0.702 & 0.580 & 0.526 & 0.206 & 0.534 & 0.890 \\
\hline & 3 Part L2 & Unigrams & 0.702 & 0.614 & 0.704 & 0.492 & 0.738 & 0.960 \\
\hline \multirow{2}{*}{$\begin{array}{l}\text { Match } \\
\text { Pyramid [22] }\end{array}$} & $\mathrm{BCE}^{*}$ & Unigrams ${ }^{\ddagger}$ & 0.475 & 0.357 & 0.599 & 0.348 & 0.682 & 0.959 \\
\hline & 3 Part L2 & Unigrams & 0.562 & 0.450 & 0.611 & 0.358 & 0.654 & 0.956 \\
\hline \multirow{2}{*}{ ARC II [10] } & Pairwise ${ }^{\ddagger}$ & Unigrams ${ }^{*}$ & 0.399 & 0.270 & 0.547 & 0.299 & 0.673 & 0.939 \\
\hline & 3 Part L2 & Unigrams & 0.465 & 0.348 & 0.577 & 0.353 & 0.671 & 0.936 \\
\hline
\end{tabular}

‡ These are the results from the best model trained using the loss and tokenization methodology presented in the original paper.

\section{CONCLUSION AND FUTURE WORK}

We presented our semantic product search model for an online retail store to improve product discovery with significant increases in KPIs. We discussed intuitions, practical tradeoffs, and key insights learned from many iterations of experiments. We introduced a 3-part hinge loss and showed that it outperforms other variants by deftly handling impressed but not purchased products. Furthermore, we showed that hashing unseen tokens improves the precision across different tokenization strategies. We observed significant improvements to offline metrics by increasing the training data and presented our data preprocessing approach to reduce training time. Finally, we presented our approach to training models across multiple GPUs to enable learning with larger embedding sizes and reduce the training time. In the future, we hope to improve the precision of our models and eliminate the need for additional heuristics to filter irrelevant results online. Our initial experiments using self-attention mechanisms and positional encodings did not show improvements in precision over our existing model, which we posit further underscores the unique nature of product search versus more traditional problems in IR and NLP. We will continue exploring approaches for scaling both training and inference.

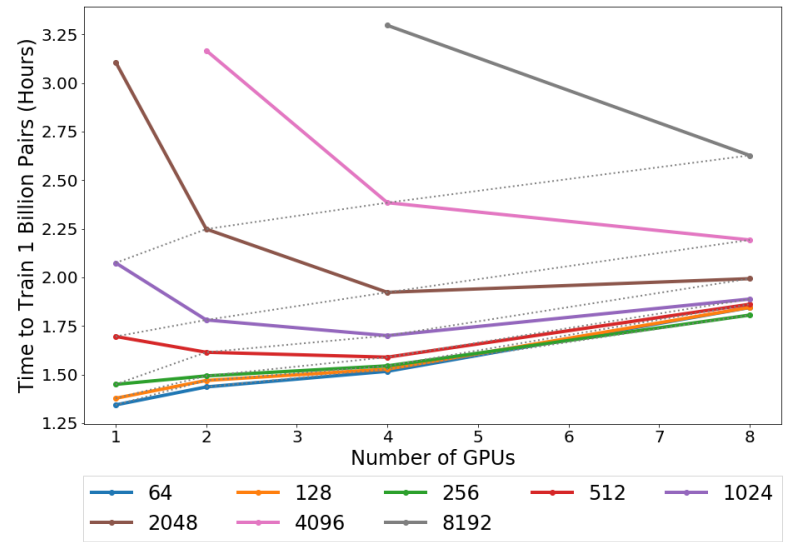

Figure 6: Training time with various embedding dimensions

\section{ACKNOWLEDGMENTS}

Nan Chen, Abhinandan Patni, and Trishul Chilimbi were instrumental in gathering data, engineering our training platform, and increasing the training speed. Yesh Dattatreya and Sunny Rajagopalan 
Table 6: Example Queries and Matched Products

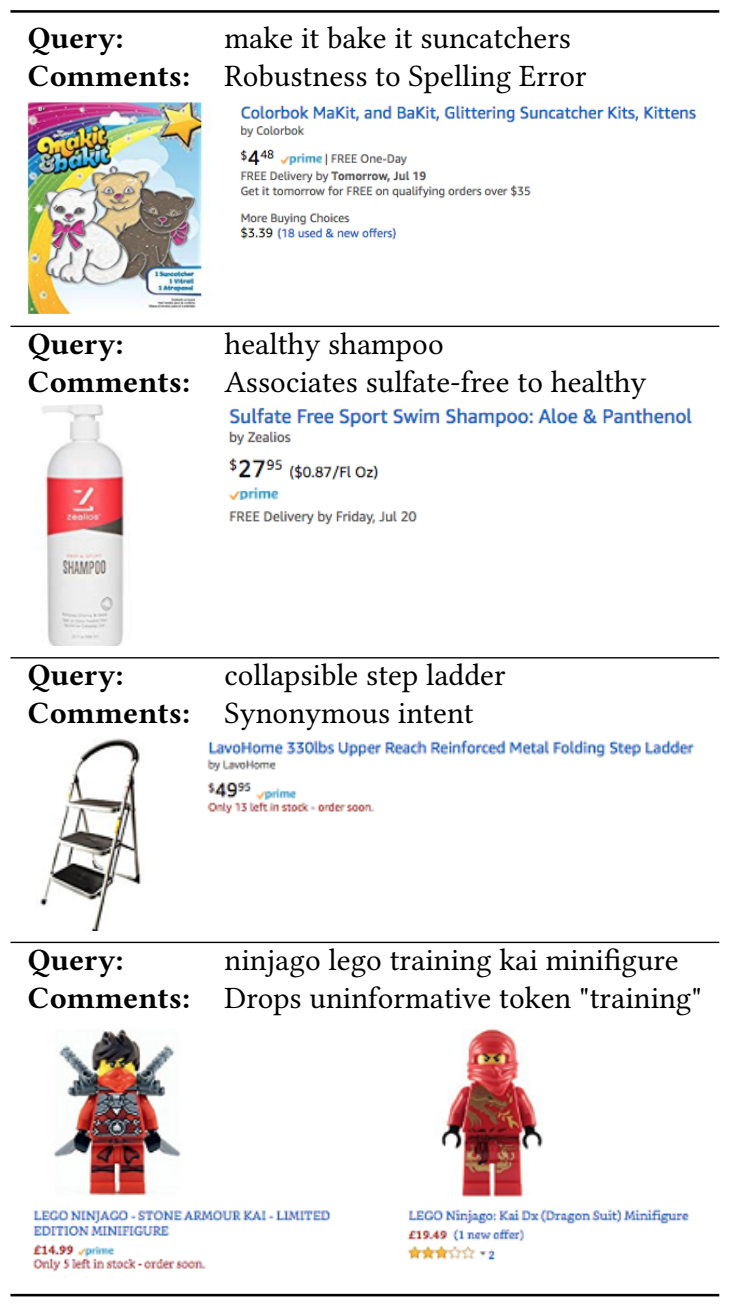

helped in hyperparameter optmization. Guy Lebanon, Vishy Vishwanathan and Inderjit Dhillon served as advisors throughout the project. Scott Le Grand and Edward Kandrot provided guidance to design and implement model parallel training.

\section{REFERENCES}

[1] Michael W Berry and Paul G Young. 1995. Using latent semantic indexing for multilanguage information retrieval. Computers and the Humanities 29, 6 (1995), 413-429.

[2] David M Blei, Andrew Y Ng, and Michael I Jordan. 2003. Latent dirichlet allocation. fournal of machine Learning research 3, Jan (2003), 993-1022.

[3] Silviu Cucerzan and Eric Brill. 2004. Spelling correction as an iterative process that exploits the collective knowledge of web users. In Proceedings of the 2004 Conference on Empirical Methods in Natural Language Processing.

[4] Hercules Dalianis. 2002. Evaluating a spelling support in a search engine. In International Conference on Application of Natural Language to Information Systems. Springer, 183-190.

[5] Scott Deerwester, Susan T Dumais, George W Furnas, Thomas K Landauer, and Richard Harshman. 1990. Indexing by latent semantic analysis. Fournal of the American society for information science 41, 6 (1990), 391-407.

[6] Fernando Diaz, Bhaskar Mitra, and Nick Craswell. 2016. Query expansion with locally-trained word embeddings. arXiv preprint arXiv:1605.07891 (2016).

[7] Susan T Dumais, Todd A Letsche, Michael L Littman, and Thomas K Landauer. 1997. Automatic cross-language retrieval using latent semantic indexing. In $A A A I$ spring symposium on cross-language text and speech retrieval, Vol. 15. 21

[8] Xavier Glorot and Yoshua Bengio. 2010. Understanding the difficulty of training deep feedforward neural networks. In In Proceedings of the International Conference on Artificial Intelligence and Statistics (AISTATS?10). Society for Artificial Intelligence and Statistics.

[9] Jiafeng Guo, Yixing Fan, Qingyao Ai, and W Bruce Croft. 2016. A deep relevance matching model for ad-hoc retrieval. In Proceedings of the 25th ACM International on Conference on Information and Knowledge Management. ACM, 55-64.

[10] Baotian Hu, Zhengdong Lu, Hang Li, and Qingcai Chen. 2014. Convolutional Neural Network Architectures for Matching Natural Language Sentences. In Proceedings of the 27th International Conference on Neural Information Processing Systems - Volume 2 (NIPS'14). MIT Press, Cambridge, MA, USA, 2042-2050. http: //dl.acm.org/citation.cfm?id=2969033.2969055

[11] Po-Sen Huang, Xiaodong He, Jianfeng Gao, Li Deng, Alex Acero, and Larry Heck. 2013. Learning deep structured semantic models for web search using clickthrough data. In Proceedings of the 22nd ACM international conference on Conference on information \& knowledge management. ACM, 2333-2338.

[12] Kai Hui, Andrew Yates, Klaus Berberich, and Gerard de Melo. 2017. Pacrr: A position-aware neural ir model for relevance matching. arXiv preprint arXiv:1704.03940 (2017)

[13] Kai Hui, Andrew Yates, Klaus Berberich, and Gerard de Melo. 2017. Re-pacrr: A context and density-aware neural information retrieval model. arXiv preprint arXiv:1706.10192 (2017)

[14] Kai Hui, Andrew Yates, Klaus Berberich, and Gerard de Melo. 2018. Co-pacrr: A context-aware neural ir model for ad-hoc retrieval. In Proceedings of the Eleventh ACM International Conference on Web Search and Data Mining. ACM, 279-287.

[15] Sergey Ioffe and Christian Szegedy. 2015. Batch normalization: Accelerating deep network training by reducing internal covariate shift. arXiv preprint arXiv:1502.03167 (2015)

[16] Armand Joulin, Edouard Grave, Piotr Bojanowski, and Tomas Mikolov. 2017. Bag of Tricks for Efficient Text Classification. In Proceedings of the 15th Conference of the European Chapter of the Association for Computational Linguistics: Volume 2, Short Papers. Association for Computational Linguistics, 427-431.

[17] CD Manning, R PRABHAKAR, and S HINRICH. 2008. Introduction to information retrieval, volume 1 Cambridge University Press. Cambridge, UK (2008).

[18] Tomas Mikolov, Ilya Sutskever, Kai Chen, Greg S Corrado, and Jeff Dean. 2013. Distributed representations of words and phrases and their compositionality. In Advances in neural information processing systems. 3111-3119.

[19] Bhaskar Mitra, Fernando Diaz, and Nick Craswell. 2017. Learning to match using local and distributed representations of text for web search. In Proceedings of the 26th International Conference on World Wide Web. International World Wide Web Conferences Steering Committee, 1291-1299.

[20] Bhaskar Mitra, Eric Nalisnick, Nick Craswell, and Rich Caruana. 2016. A dual embedding space model for document ranking. arXiv preprint arXiv:1602.01137 (2016).

[21] Hamid Palangi, Li Deng, Yelong Shen, Jianfeng Gao, Xiaodong He, Jianshu Chen, Xinying Song, and Rabab Ward. 2016. Deep sentence embedding using long short-term memory networks: Analysis and application to information retrieval. IEEE/ACM Transactions on Audio, Speech and Language Processing (TASLP) 24, 4 (2016), 694-707.

[22] Liang Pang, Yanyan Lan, Jiafeng Guo, Jun Xu, Shengxian Wan, and Xueqi Cheng. 2016. Text Matching as Image Recognition.. In AAAI. 2793-2799.

[23] Ruslan Salakhutdinov and Geoffrey Hinton. 2009. Semantic hashing. International fournal of Approximate Reasoning 50, 7 (2009), 969-978.

[24] Yelong Shen, Xiaodong He, Jianfeng Gao, Li Deng, and Grégoire Mesnil. 2014. A latent semantic model with convolutional-pooling structure for information retrieval. In Proceedings of the 23rd ACM International Conference on Conference on Information and Knowledge Management. ACM, 101-110.

[25] Grigori Sidorov, Francisco Velasquez, Efstathios Stamatatos, Alexander Gelbukh, and Liliana Chanona-Hernández. 2014. Syntactic n-grams as machine learning features for natural language processing. Expert Systems with Applications 41, 3 (2014), 853-860.

[26] Shengxian Wan, Yanyan Lan, Jun Xu, Jiafeng Guo, Liang Pang, and Xueqi Cheng. 2016. Match-srnn: Modeling the recursive matching structure with spatial rnn. arXiv preprint arXiv:1604.04378 (2016).

[27] Xing Wei and W Bruce Croft. 2006. LDA-based document models for ad-hoc retrieval. In Proceedings of the 29th annual international ACM SIGIR conference on Research and development in information retrieval. ACM, 178-185.

[28] Kilian Weinberger, Anirban Dasgupta, Josh Attenberg, John Langford, and Alex Smola. 2009. Feature hashing for large scale multitask learning. arXiv preprint arXiv:0902.2206 (2009).

[29] Liu Yang, Qingyao Ai, Jiafeng Guo, and W Bruce Croft. 2016. aNMM: Ranking short answer texts with attention-based neural matching model. In Proceedings of the 25th ACM International on Conference on Information and Knowledge Management. ACM, 287-296.

[30] Justin Zobel and Alistair Moffat. 2006. Inverted files for text search engines. ACM computing surveys (CSUR) 38, 2 (2006), 6. 
Table 7: Shared versus Decoupled Embeddings for Query and Product

\begin{tabular}{|c|c|c|c|c|c|c|c|c|}
\hline Tokenization & Loss & Shared & Recall & MAP & $\begin{array}{l}\text { Matching } \\
\text { NDCG }\end{array}$ & $\begin{array}{l}\text { Matching } \\
\text { MRR }\end{array}$ & $\begin{array}{c}\text { Ranking } \\
\text { NDCG }\end{array}$ & $\begin{array}{c}\text { Ranking } \\
\text { MRR }\end{array}$ \\
\hline \multirow{2}{*}{ Unigrams } & \multirow{2}{*}{$\mathrm{BCE}$} & $\mathrm{F}$ & 0.520 & 0.418 & 0.649 & 0.420 & 0.692 & 0.953 \\
\hline & & $\mathrm{T}$ & 0.586 & 0.486 & 0.695 & 0.473 & 0.711 & 0.954 \\
\hline \multirow{2}{*}{ Unigrams } & \multirow{2}{*}{ MSE } & $\mathrm{F}$ & 0.131 & 0.069 & 0.389 & 0.307 & 0.690 & 0.956 \\
\hline & & $\mathrm{T}$ & 0.238 & 0.144 & 0.490 & 0.377 & 0.680 & 0.948 \\
\hline \multirow{2}{*}{ Unigrams } & \multirow{2}{*}{2 Part L2 } & $\mathrm{F}$ & 0.622 & 0.553 & 0.773 & 0.581 & 0.775 & 0.974 \\
\hline & & $\mathrm{T}$ & 0.651 & 0.576 & 0.768 & 0.549 & 0.776 & 0.973 \\
\hline \multirow{2}{*}{ Unigrams } & \multirow{2}{*}{3 Part L2 } & $\mathrm{F}$ & 0.730 & 0.662 & 0.771 & 0.593 & 0.766 & 0.972 \\
\hline & & $\mathrm{T}$ & 0.735 & 0.664 & 0.791 & 0.591 & 0.772 & 0.973 \\
\hline \multirow{2}{*}{$\begin{array}{l}\text { Unigrams+Bigrams+ } \\
\text { Char Trigrams+OOV }\end{array}$} & \multirow{2}{*}{3 Part L2 } & $\mathrm{F}$ & 0.781 & 0.739 & 0.799 & $0.639^{*}$ & 0.784 & 0.980 \\
\hline & & $\mathrm{T}$ & $0.790^{*}$ & $0.743^{*}$ & $0.805^{*}$ & 0.625 & $0.794^{*}$ & $0.981^{*}$ \\
\hline
\end{tabular}

Table 8: Impact of Out-of-Vocabulary Bin Size

\begin{tabular}{l|cccc|cc}
\hline Tokenization & Recall & MAP & $\begin{array}{c}\text { Matching } \\
\text { NDCG }\end{array}$ & $\begin{array}{c}\text { Matching } \\
\text { MRR }\end{array}$ & $\begin{array}{c}\text { Ranking } \\
\text { NDCG }\end{array}$ & $\begin{array}{c}\text { Ranking } \\
\text { MRR }\end{array}$ \\
\hline Unigrams+Bigrams+Char Trigrams & 0.764 & 0.707 & 0.800 & 0.615 & 0.794 & 0.978 \\
Unigrams+Bigrams+Char Trigrams+5K OOV & 0.767 & 0.711 & 0.802 & 0.617 & 0.800 & 0.979 \\
Unigrams+Bigrams+Char Trigrams+10K OOV & 0.774 & 0.714 & 0.811 & 0.633 & 0.804 & 0.979 \\
Unigrams+Bigrams+Char Trigrams+50K OOV & 0.777 & 0.725 & 0.810 & $\mathbf{0 . 6 3 7}$ & $\mathbf{0 . 8 0 7}$ & 0.981 \\
Unigrams+Bigrams+Char Trigrams+100K OOV & 0.784 & 0.733 & $\mathbf{0 . 8 1 7}$ & 0.629 & $\mathbf{0 . 8 0 7}$ & $\mathbf{0 . 9 8 2}$ \\
Unigrams+Bigrams+Char Trigrams+250K OOV & $\mathbf{0 . 7 9 0}$ & 0.740 & 0.814 & 0.623 & 0.804 & 0.980 \\
Unigrams+Bigrams+Char Trigrams+500K OOV & $\mathbf{0 . 7 9 0}$ & $\mathbf{0 . 7 4 3}$ & 0.805 & 0.625 & 0.794 & 0.981 \\
\hline
\end{tabular}

\section{A ADDITIONAL EXPERIMENTS}

This section details additional experiments completed to determine the model architecture and to tune model hyperparameters.

We demonstrate empirically in Table 7 that sharing the embedding layer between the query and product arm tends to perform better for matching results across multiple tokenizations and loss functions. As we described previously, sharing the embedding layer helps local word-level matching and generalization to unseen tokens when using OOV bins. Note that in this experiment, the number of model parameters was held constant. So the embedding dimension was 256 for the shared embedding layer but 128 for each of the decoupled query and product embedding layers.

In Table 8, we present the results of varying the OOV bin size. We see that matching performance improves as the bin size increases, although ranking performance peaks at lower bin sizes. These results confirm the intuition that adding OOV hashing leads to generalization to unseen tokens. This generalization improves matching performance as there are fewer spurious matches resulting from OOV tokens mapping to the same bucket and/or simply excluding OOV tokens. 